\title{
On the conditions for the determination of the thermal properties of flowing gases by using oscillatory thermal excitation
}

\author{
Diego F. Reyes-Romero ${ }^{1}$, Gerald A. Urban ${ }^{2}$ \\ ${ }^{1}$ Innovative Sensor Technology AG, Stegrütistrasse 14, 9642 Ebnat-Kappel, Switzerland, \\ diego.reyes@ist-ag.com \\ ${ }^{2}$ University of Freiburg, George-Koehler-Allee 103, Freiburg, Germany
}

\begin{abstract}
This contribution presents the necessary conditions for the determination of the thermal properties of a gas in a channel under flowing conditions. The measurement principle relies on the modulation of the penetration depth of harmonic thermal waves by means of the oscillation frequency. Harmonic waves produced on the wall of the flow channel are confined within flow boundary layer that forms over the surface of the channel's wall. Simulations and experiments using gases show that thermal conductivity and the volumetric heat capacity can be determined under flow conditions if $v /(D \omega)<1$, where $v$ is the average flow speed in the channel, $\omega$ the frequency and $D$ the channel diameter. This verifies the results reported in the literature for liquids only.
\end{abstract}

Key words: thermal conductivity, thermal flow sensor, harmonic excitation

\section{Introduction}

Determination of the thermal properties of flowing gases raises a lot interest in applications where the quality or composition of the gas must be assessed without stopping the flow. Such applications include natural gas, contaminants monitoring among others.

Usual approaches involve the use of complex structures with which is possible to measure the thermal properties in no-flow conditions. For instance, a dead volume with several micromachined bridge-structures were used by van Baar et al. [1] to determine the thermal conductivity $k$ without the influence of the flow rate. A steady heat signal of known power is applied to a heater and its temperature rise is used as a measure of the thermal conductivity.

A similar approach uses a cylinder inside a flow channel. The end facing the flow has a small opening allowing the fluid to be trapped inside the cylinder. Then, without disturbances, a hotwire is used to measured thermal conductivity [2]. Another usual solution is to have a low flow bypass [3]. Inside this bypass, valves stop the flow and a thermal sensor determines the thermal properties of the gas.

In order to avoid interrupting the flow, an interesting approach proposes four independent, 500- $\mu \mathrm{m}$-long platinum structures called thermal conductivity detectors that are suspended successively in the middle of the channel. This structure is capable of achieving a very high precision: $260 \mathrm{ppb}$ of hexane in helium [4]. These detectors are independently controlled so that the temperature distribution in the second and third structures is constant. Thus, the thermal conductivity is determined regardless of the flow velocity by measuring the heat dissipated by the second and the third resistors.

The aforementioned approaches do not allow for the determination of the volumetric heat capacity $\rho c_{p}$. For this, dynamic thermal excitation is necessary. Beigelbeck [5] have successfully used harmonic thermal excitation to determine the thermal conductivity and thermal diffusivity (hence, the volumetric heat capacity) of liquids of known speed. For this, amplitude and phase information of the temperature oscillations is fitted over a wide range of frequencies.

A similar method is the so-called $3 \omega$ method, which also relies on measuring the harmonic temperature oscillations produced by a single thermistor. This method has bees successfully used in liquids [6], and, more recently in gases [7], to determine $k$ and $\rho c_{p}$. 
Hong and Kim [8] succeeded using the $3 \omega$ method to measure the thermal conductivity of liquids flowing in circular pipes. The established the necessary conditions for determine the conductivity of liquids. This has not been extended to gases yet.

In this contribution we explore the conditions that have to fulfill in order to measure the thermal conductivity and volumetric heat capacity of flowing gases. For this, the excitation method would rely in harmonic heat generation.

\section{Theoretical Background}

Hong and Kim [8] found that the thermal conductivity of the fluid could be determined regardless of its flow rate. They achieved this by setting frequency sweep at high values because increasing the frequency $\omega$ modulates the thickness of the thermal boundary layer to be much thinner than the momentum boundary layer.

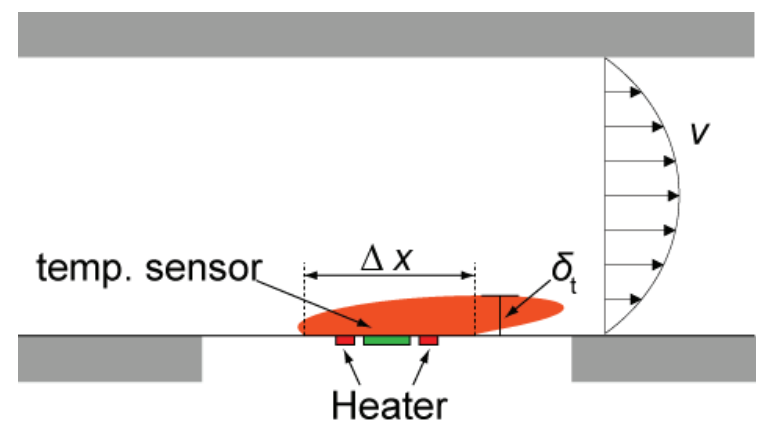

Figure 1. Modulation of the thermal penetration depth with the frequency. Most heat transfer is confined to a low-flow region close to the wall surface.

The penetration depth for harmonic thermal waves produced by a long, thin heater is given by [9]

$\delta_{\mathrm{t}}=\sqrt{\frac{k}{\omega \rho c_{\mathrm{p}}}}$.

As shown in Figure 1, a the harmonic thermal boundary layer can be brought down to a region close to the channel wall where, due to the nonslip condition, the local flow velocity is close to zero. Hence, the flow velocity does not affect most part of the thermal exchange between the heater and the fluid. However, if the average flow rate $v$ is increased above a certain threshold, the flow profile flattens out over the channel's wall, which would vanish this flowindependence. For liquids only, Hong and Kim [8] found this threshold to be

$$
\frac{2 v}{D \omega}<2.5 \text {, }
$$

where $D$ is the diameter of the pipe, $v$ the average velocity and $\omega$ the angular frequency. They defined this criterion for a maximum variation of $1 \%$ in the measurement variables.

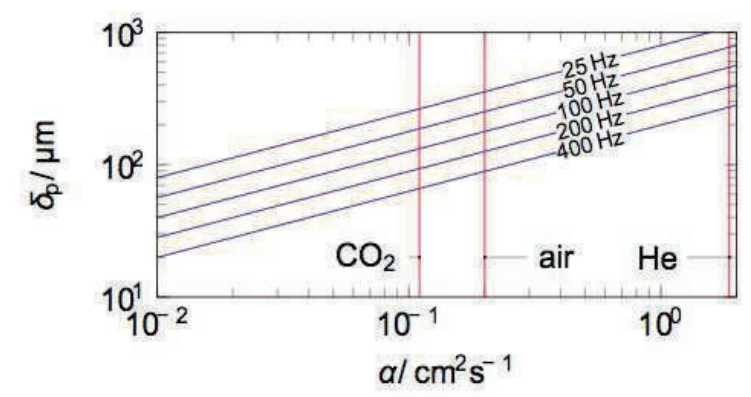

Figure 2. Penetration depth in terms of the frequency and thermal difussivity $\left(\alpha=k / \rho c_{p}\right)$. Some common gases are depicted.

Although Equation (2) was verified by simulations for water and ethanol, and experimentally only for water, Hong and Kim claim that it might be valid for other fluids, including gases. They swept the frequency between $1.5 \mathrm{~Hz}$ and $12.7 \mathrm{~Hz}$ and the total deviation between flow and no-flow conditions in the measurement of $k$ is just $2.3 \%$ for flow velocities up to $2.47 \mathrm{~m} / \mathrm{s}$ (about $560 \mathrm{sccm}$ ). The average heating power is $P=3.25 \mathrm{~mW}$. The major drawback of the $3 \omega$ method is that it requires sweeping the frequency. This operation could take too long depending on how broad the frequency sweep is.

Figure 2 presents the expected thickness of the thermal boundary layer for gases based on the range of possible thermal diffusivities of gases; for air, $\delta_{\mathrm{t}}<100 \mu \mathrm{m}$ only when $f>400 \mathrm{~Hz}$. So, for channels in the millimeter range, the oscillation frequency has to be relatively high (over $100 \mathrm{~Hz}$ ).

\section{Measurement Principle}

The measurement principle proposed in this work is not based on the $3 \omega$ method. It uses steady harmonic generation at a constant, welldefined frequency $f$. This reduces the measuring time with respect to the $3 \omega$ method since no frequency sweep is required. For this, at least one heater and one temperaturesensing element are required.

Not only the thickness of the thermal boundary layer shrinks with increasing frequency but also the region around the heater (denoted by $\Delta \mathrm{x}$ in Figure 1). Heat transfer inside this region is only affected by the thermal properties of the gas and the sensor regardless of the flow velocity.

For this reason, a heater structure that closely surrounds the temperature sensor (as shown in Figure 1) would improve the measured signals. A micromachined thermal flow sensor was 
fabricated accordingly (see Figure 3 ). The sensor die, whose dimensions are $12.7 \mathrm{~mm} x$ $12.7 \mathrm{~mm} \times 5.25 \mathrm{~mm}$, features a 1.5 - $\mu \mathrm{m}$-thick, $\mathrm{SiO}_{2}-\mathrm{SIN}_{\mathrm{x}}$ membrane. Five amorphous germanium thermistors (with a $2.8 \% / \mathrm{K} \mathrm{TCR}$ ) are arranged to allow for two-dimensional flow measurement. Since the heater closely surrounds the central thermistor (CT), the latter always lies within penetration depth, thus enabling the determination of the thermal properties of the fluid, namely, $k$ and $\rho c_{p}$.

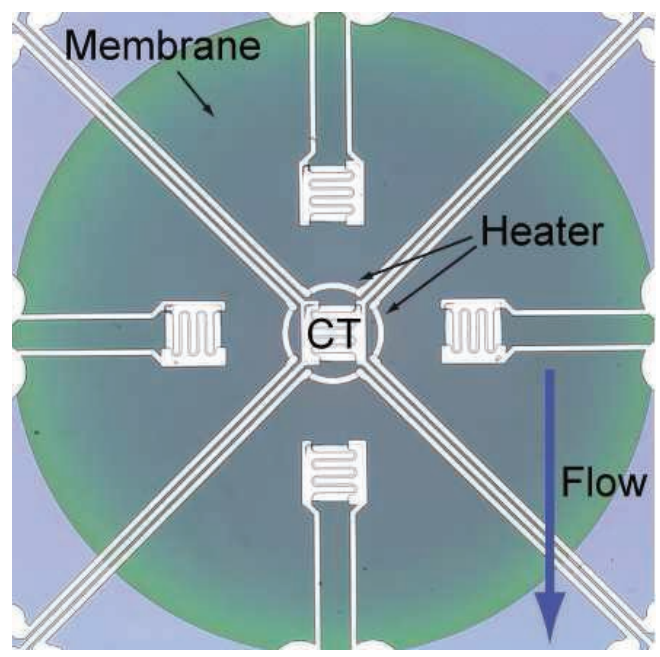

Figure 3. Close-up of the membrane of the sensor. A 1-mm-diameter membrane holds a germanium thermistor (CT), which is closely surrounded by a chromium heater.

Since the heating frequency is constant, the measuring variables are defined as (a) the amplitude of the temperature harmonic oscillation measured at the central thermistor, $\theta_{\mathrm{CT}}$; and (b) the phase difference between the temperature harmonic oscillation measured at the central thermistor and harmonic heat generation produced at the heater, $\varphi_{\mathrm{CT}}$. The these two variables are functions of the thermal properties of the gas, that is,

$\left|\theta_{\mathrm{CT}}\right|=f\left(k, \rho c_{\mathrm{p}}\right), \varphi_{\mathrm{CT}}=g\left(k, \rho c_{\mathrm{p}}\right)$

\section{Simulations}

The simplified two-dimensional geometry shown in Figure 4 was used to simulate the simulated the general behavior of the flow sensor in the frequency domain. The model considers a gas flow channel, a membrane and a chamber where the gas flow is zero. Flow is kept in laminar regime, thus a parabolic profile is modeled in the flow channel. The same gas occupies the regions above and below the membrane. The width of the channel is assumed to be much larger than its height $(H)$; therefore, the equivalent hydraulic diameter is $D=H / 2$

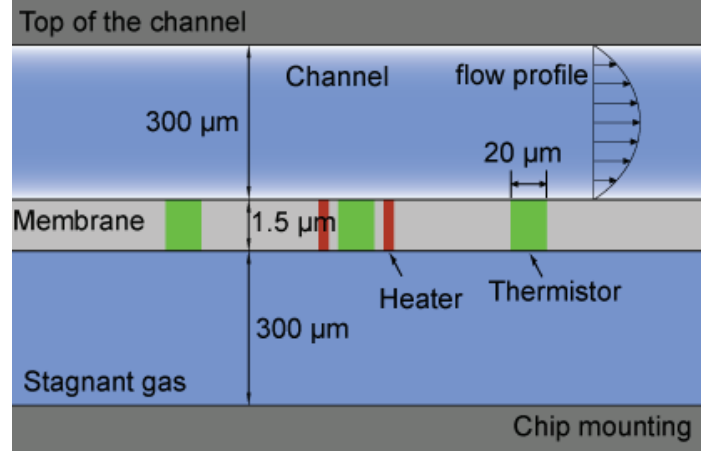

Figure 4. Two-dimensional simulation model

The model was simulated for frequencies between $0.1 \mathrm{~Hz}$ and $400 \mathrm{~Hz}$ and average flow speed between $0.01 \mathrm{~m} / \mathrm{s}$ and $5 \mathrm{~m} / \mathrm{s}$. Figure 5 summarizes the relation between the oscillations in temperature measured by the central thermistor and the magnitude $2 v / D \omega$ (or $v / H \omega)$. Amplitude $\left|\theta_{\mathrm{CT}}\right|$ and phase $\varphi_{\mathrm{CT}}$ are both normalized by the corresponding value $\left(\left|\theta_{\mathrm{CT}, 0}\right|\right.$, $\left.\varphi_{C T}\right)$ obtained in no-flow conditions $(v=0)$. The deviation on the amplitude of the thermal oscillations is less than $1 \%$ if $v / H \omega<1$ and $v / H \omega<2$ for air and helium respectively. For the phase shift, the variation is less than $1 \%$ if $v / H \omega<2$ for both gases.
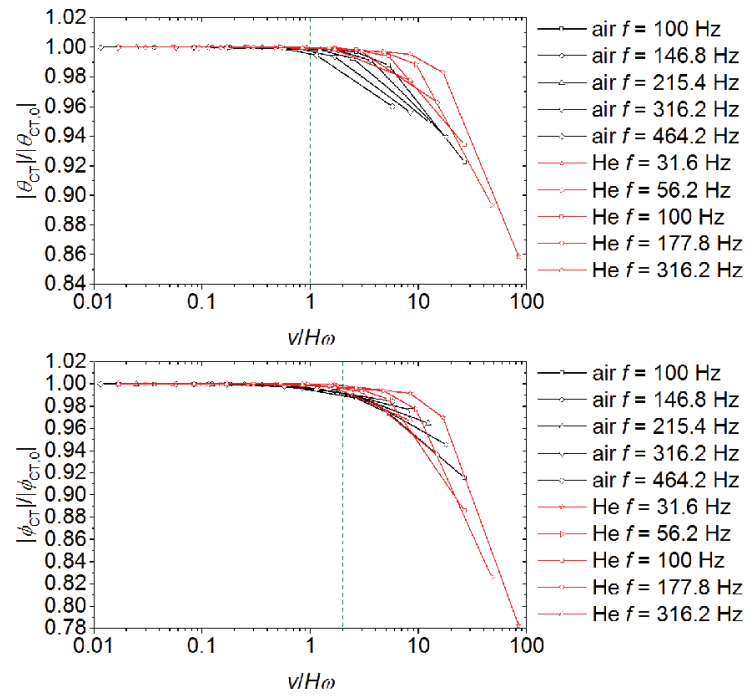

Figure 5. Normalized amplitude (top) and normalized phase shift (bottom) of the temperature oscillations at the central thermistor for air (blue) and helium (red) versus the magnitude $2 \mathrm{v} / \mathrm{D} \omega(\mathrm{v} / \mathrm{H} \omega)$

For the normalized amplitude, the critical value of $v / H \omega$ seems to depend on the thermal diffusivity of the gas. This results from weaker attenuation and larger penetration depth that thermal waves experience for gases with larger thermal diffusivity.

These results on gases seem to agree with what Hong and Kim found for water and ethanol (Equation 2). Hence, the criterion for flow 
independency in the measurement of the thermal properties is proposed to be

$\frac{v}{H \omega}<2$.

In this case, the measurement variables $\left|\theta_{\mathrm{CT}}\right|$ and $\varphi_{\mathrm{CT}}$ are independent of the average flow velocity and can be used to determine the thermal properties of a flowing fluid. This condition applies for gases measured with the sensor presented in Figure 1, that is, with a central heater-thermistor structure.

According to Equation (4) and the dimensions of the channel in Figure 4, the maximum average velocity in the channel for a $1 \%$ error at $200-\mathrm{Hz}$ excitation is $0.75 \mathrm{~m} / \mathrm{s}$.

\section{Measurement Setup}

The experimental setup consists of PMMA rectangular channel. Its cross-section is $14 \mathrm{~mm}$ $x 0.6 \mathrm{~mm}$. Sensor depicted in Figure 3 is embedded in the channel's floor. A mass flow controller (MFC) regulates the gas flow rate inside from a compressed bottle into the channel. The flow speed can be regulated between 20 and $1000 \mathrm{sccm}$. A voltage source drives the heater with a steady sinusoidal signal. The resulting heat signal oscillates at $200 \mathrm{~Hz}$ with an average value of $0.5 \mathrm{~mW}$. The resistance of the thermistor is measured with a current-to-voltage converter. The output voltage is digitally acquired.

\section{Experimental Results}

Hong and Kim [8] proposed that if $v / H \omega<2.5$, the thermal conductivity of flowing liquids can be determined by means of the $3 \omega$ method. Simulations in this paper suggest that the determination of the thermal properties of flowing gases is possible if $v / H \omega<2$. In this case, unlike the $3 \omega$ method, the proposed measurement procedure only requires a single operating frequency and not a whole frequencysweep.

Over 180 experimental measurement points of $\omega$ and $v$ are depicted in Figure 6 in order to show the experimental relationship between the number $v / H \omega$ and the measurement magnitudes. Amplitude and phase are normalized by the corresponding value $\left(\left|\theta_{\mathrm{CT}, 0}\right|\right.$ and $\left.\varphi_{\mathrm{CT}, 0}\right)$ obtained with the lowest possible flow rate at the same $\omega$ for each gas. Data points include oscillation frequencies between $40 \mathrm{~Hz}$ and $500 \mathrm{~Hz}$. Experimental results agree well with the simulation results (see Figure 5). Variation of the amplitude and phase signals is under $1 \%$ for $v / H \omega<2$ and $v / H \omega<1$, respectively. Only helium does not meet the criterion. Due to helium's low density, at the lowest flow rates, the much denser atmospheric air is able to penetrate the channel through the outlet and displace the much lighter helium. This might also explain the fact that, for helium, data points do not form a plateau for low values of $v / H \omega$.
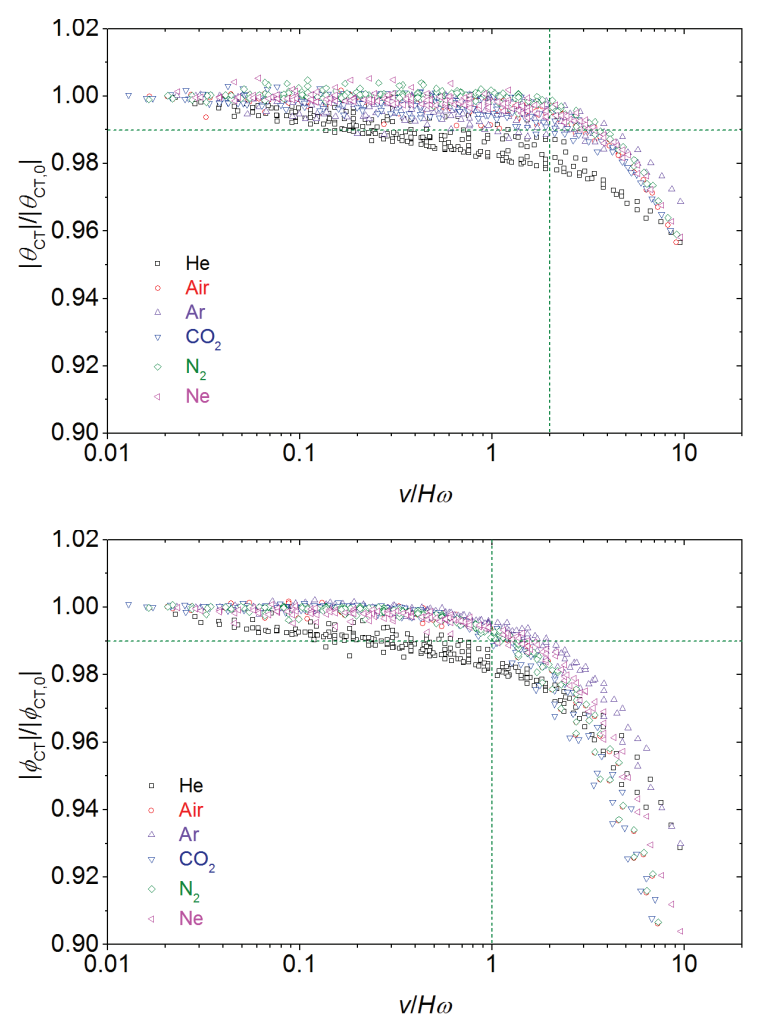

Figure 6. Normalized amplitude (top) and normalized phase shift (bottom) of the measured temperature oscillations at the central thermistor for various pure gases versus $v / H \omega$. Green lines mark the critical values for a variation of $1 \%$ in the measurement signals

\section{Conclusion}

Table 1 summarizes the simulated and experimental critical values of $\mathrm{v} / \mathrm{H \omega}$ for a maximum variation of $1 \%$ in the measurement signals. These values seem to agree well with each other and in particular to the value reported by Hong and $\mathrm{Kim}$ [8] for liquids (Equation 2). However, results in Table 1 suggest that the criterion should be smaller for gases, as described by Equation (4).

Table 1. Critical value of $\mathrm{v} / \mathrm{H} \omega$ for a maximal variation of $1 \%$ in the measurement signal obtained from simulations and experimentally

\begin{tabular}{ccc}
\hline Signal & Simulation & Experiment \\
\hline Amplitude $\left|\theta_{\mathrm{CT}}\right|$ & 1 & 2 \\
\hline Phase $\varphi_{\mathrm{CT}}$ & 2 & 1 \\
\hline
\end{tabular}


According to this criterion, for the channel used in the experiments and a frequency of $200 \mathrm{~Hz}$, the maximum allowed average flow speed that guarantees a $1 \%$ error is $1.5 \mathrm{~m} / \mathrm{s}$. This corresponds to a flow rate of $760 \mathrm{sccm}$. As a result, is possible to measure the thermal conductivity and the volumetric heat capacity of gases using the sensor in Figure 3. The measurement principle for achieving this goal is better described in Reference [10].

\section{References}

[1] J. J. van Baar, R. J. Wiegerink, T. S. J. Lammerink, G. J. M. Krijnen, and M. Elwenspoek. Micromachined structures for thermal measurements of fluid and flow parameters. Journal of Micromechanics and Microengineering, 11(4), pp. 311-318, July 2001.

[2] W. Schrittenlacher. Thermal flow-rate sensor and method for determining the flow rate of a fluid, 2003. US Patent 6536273 B2.

[3] M. Grinstein, J. Y. Mantinband, S. Bentov, and M. Adler. Flow rate meter, 2013. US Patent Application US 20130041234 A1.

[4] B. Kaanta, A. Jonca, H. Chen, and X. Zhang. Temperature distribution on thermal conduc- tivity detectors for flow rate insensitivity. Sensors and Actuators A: Physical, 167(2), pp. 146-151, June 2011.

[5] Beigelbeck, R., Nachtnebel, H., Kohl, F., \& Jakoby, B. A novel measurement method for the thermal properties of liquids by utilizing a bridgebased micromachined sensor. Measurement Science and Technology, 22(10), p. 105407, 2011

[6] Moon, I. K., Jeong, Y. H., \& Kwun, S. I., The $3 w$ technique for measuring dynamic specific heat and thermal conductivity of a liquid or solid. Review of Scientific Instruments, 67(1), p. 29, 1996.

[7] Gauthier, S., Giani, A., \& Combette, P., Gas thermal conductivity measurement using the three-omega method. Sensors and Actuators A: Physical, 195, pp. 50-55, 2013.

[8] J. Hong and D. Kim. Measuring the Thermal Conductivity of Flowing Liquid Samples Using the Three Omega Method. Journal of Heat Transfer, 134(9), p. 094502, 2012.

[9] D. G. Cahill. Thermal conductivity measurement from 30 to $750 \mathrm{~K}$ : the $3 \omega$ method. Review of Scientific Instruments, 61(2), p. 802, 1990.

[10] Reyes, D. F., Kogan, K., Cubukcu, A. S., \& Urban, G. A, Simultaneous Flow and Thermal Conductivity Measurement of Gases Utilizing a Calorimetric Flow Sensor. Sensors and Actuators A: Physical, 203, pp. 225-233, 2013. 